\title{
Juvenile hormone: Production, regulation, current application in vector control and its future applications
}

\author{
Nur Aliah, N.A. ${ }^{1}$, Ab-Rahim, S. ${ }^{2}$, Moore, H.E. ${ }^{3}$, Heo, C.C. ${ }^{1,4^{*}}$ \\ ${ }^{1}$ Department of Medical Microbiology and Parasitology, Faculty of Medicine, Universiti Teknologi MARA Selangor Branch, Sungai Buloh Campus, \\ 47000 Sungai Buloh, Selangor, Malaysia \\ ${ }^{2}$ Department of Biochemistry and Molecular Medicine, Faculty of Medicine, Universiti Teknologi MARA Selangor Branch, Sungai Buloh Campus, \\ 47000 Sungai Buloh, Selangor, Malaysia \\ ${ }^{3}$ Cranfield Forensic Institute, Cranfield University, Shrivenham, Swindon, SN6 8LA UK \\ ${ }^{4}$ Institute for Pathology, Laboratory and Forensic Medicine (I-PPerForM), Universiti Teknologi MARA Selangor Branch, Sungai Buloh Campus, \\ 47000 Sungai Buloh, Selangor, Malaysia \\ *Corresponding author: chin@uitm.edu.my
}

\section{ARTICLE HISTORY}

Received: 17 March 2021

Revised: 9 June 2021

Accepted: 9 June 2021

Published: 31 July 2021

\begin{abstract}
Juvenile hormone is an exclusive hormone found in insects which involves regulating various insect physiology. A total of eight juvenile hormones have been identified in insects which include JH 0, JH I, JH II, JH III, 4-methyl JH I (Iso- JH 0), JHB III, JHSB III, and MF. Corpora allata are the glands responsible for the production and synthesis of these hormones. They are involved in moulting, reproduction, polyethism, and behavioural regulations in different orders of insects. Factors such as diet temperatures, photoperiods, and plant compounds affect the biosynthesis and regulation of juvenile hormones. Juvenile hormones analogue is usually used to disrupt normal regulation of $\mathrm{JH}$ and this analogue is categorized as insect-growth regulators (IGRs) and is widely used in pest control as an alternative to chemical insecticides. Other applications of biosynthesis activities of this hormone have not been explored in the area of JHs. In this review, current applications of JHs with an addition of their future application will be discussed.
\end{abstract}

Keywords: Juvenile hormones; corpora allata; insect brain; insect physiology; pest control.

\section{INTRODUCTION}

In 1936, an insect "inhibitory hormone" or currently known as juvenile hormones (JHs) was first discovered by Sir V. B. Wigglesworth in Rhondius prolixus (Hemiptera: Triatominae). In this early discovery, JH is classified as a sesquiterpenoid hormones found in insect. It is secreted and synthesis by a pair of endocrine glands located near the tritocerebrum area of an insect brains, known as corpora allata (CA). Secretion and biosynthesis of JHs by the CA regulate various physiological roles in insects such as reproduction, development, behaviour, and caste determination. Nevertheless, $\mathrm{JHs}$ are well known in their roles in metamorphosis and reproduction where inhibition of adult moulting and yolk deposition in eggs are regulated according to the level of JHs in the haemolymph (Gullan \& Cranston, 2010). To date, eight different types of $\mathrm{JHs}$ have been identified in insects,

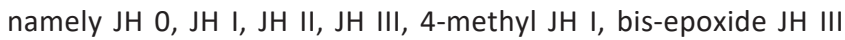
(JHB III), skipped bis-epoxide (JHSB III), and methyl farnesoate (MF). JH homologs present in various insects are summarised in Table 1.

Usually, mixtures of $\mathrm{JHs}$ are found in insects with a homologous function in different insect orders (Gullan \&
Cranston, 2010). These hormones are usually found in a mixture of different $\mathrm{JHs}$ but the most common is $\mathrm{JH} \mathrm{III} \mathrm{and} \mathrm{it}$ can be found in Lepidoptera, Diptera, and Hymenoptera. A mixture of $\mathrm{JH} 0, \mathrm{JH} \mathrm{I}$, and $\mathrm{JH} \mathrm{III} \mathrm{are} \mathrm{found} \mathrm{in} \mathrm{Lepidoptera} \mathrm{only}$ (i.e., Manduca sexta, Hyalophora cecropia). However, during the embryonic stage of $M$. sexta, only a mixture of $\mathrm{JH} \mathrm{O}$ and $\mathrm{JH}$ I were found and there were no titres of JH III observed (Bergot et al., 1980). This may be related to different biosynthesis and regulation of $\mathrm{JH}$ during early stage of insect development. Occurrence of $\mathrm{JH}$ I were rarely reported in insects compared to JH III but in 2004, JH III and JH I were identified in the Mediterranean field cricket, Gryllus bimaculatus (Orthoptera: Gryllidae). In the same study, JH I, JH II, and JH III were found concurrently in the fifth instar larvae of the fall armyworm, Spodoptera frugiperda (Noctuidae) (Westerlund \& Hoffmann, 2004).

In Diptera, one exclusive JH homologs known as bisepoxide JH III (JHB III) was discovered in Drosophila melanogaster (Drosophilidae), Phormia regina (Calliphoridae), Aedes aegypti (Culicidae), Neobellieria bullata (Sarcophagidae), Calliphora vomitoria (Calliphoridae), and Lucilia cuprina (Calliphopridae). This discovery was reviewed by Yin (1994) where JHB III were identified with two epoxy groups in JH 
structure instead of one epoxy group like other $\mathrm{JH}$ homologs. A higher rate of JHB III biosynthesis compared to JH III was found in Diptera, indicating that $\mathrm{JH}$ III is not the sole $\mathrm{JH}$ in this Order (Bylemans et al., 1998). Nevertheless, the specific roles of this $\mathrm{JH}$ homolog are still unknown.

$\mathrm{JHs}$ are classified as a true hormone in insects, but the discovery of methyl farnesoate (MF), which is a hormone found in crustaceans, is believed to be grouped together with $\mathrm{JHs}$ because of their function. However, some believed that MF is not related to JHs. In crustaceans, MF serve as hormones in development and reproduction regulation similar to $\mathrm{JH}$ in insects. In a recent study by Di-Wen et al. (2015), MF is reported to act as an immediate precursor and hormone in the regulation of metamorphosis in $D$. melanogaster. In terms of the structure, MF is also classified as sesquiterpenoid compounds, but lacking the epoxide group. In order to prove the identity of MF, further structural and functional studies need to be conducted for a definite classification.

This review will briefly discuss about JH production, regulation, and their applications of $\mathrm{JH}$ is pest and vector control, developmental studies as well as future applications.

Juvenile hormone regulation by corpora allata and accessory glands $\mathrm{JH}$ is known to be produced and synthesised by a pair of glands named CA. This gland can be categorized into five difference morphological types according to their positions which are: (1) lateralized; (2) distally lateralized; (3) semi centralized; (4) centralized; and (5) annular types (Goodman \& Cusson, 2012)

However, the role of CA as the only organ to regulate the secretion and synthesis of $\mathrm{JH}$ is proven to be inaccurate by various researchers (Tobe et al., 1985; Bylemans et al., 1998). A concise review on the regulation of $\mathrm{CA}$ in $\mathrm{JH}$ synthesis and regulation has been done by K. Hartfelder (2000). Based on a study by Wu et al. (1987), decapitation of $D$. melanogaster brain has resulted in the activation of CA to secrete JH. The absent of the brain significantly affect JH production in this species. Also, the absent of the brain affect the hormonal control in the reproduction of $D$. melanogaster where yolk peptides failed to be synthesized. This finding is further supported by the radiochemical assay (RCA) performed on CA attached to the brain (Bylemans et al., 1998). Attachment of the brain to the $\mathrm{CA}$, known as Br-CA complex, was observed to be more productive in synthesizing $\mathrm{JH}$ than the one without the brain.

In the absent of the brain, allatotropin will be produced by accessory glands, fat body, ovaries, and the gut. Allatotropin is a peptide that stimulates the secretion of $\mathrm{JH}$ to regulate the hormonal control. The effect of allatotropin on CA has been demonstrated by the application of synthetic allatotropin of $M$. sexta to the CA of Lacanobia oleracea (Lepidoptera: Noctuidae) where a stimulation by $37 \%$ was observed in the CA of L. oleracea (Audsley et al., 1999). In the same study, a peptide that inhibit CA activity known as allatostatin was also applied on the same insect and a maximum inhibition by $54 \%$ was recorded. However, applications of synthetic allatostatin and allatotropin did not show the same affect among insect orders, for example, cockroach's allatostatin, Dip- allatostatin-2, had no significant affect on JH synthesis on L. oleracea when compared to $M$. sexta. These findings suggested that a diversity of allotostatin and allatotropin may be present among the orders of insect. Nonetheless, the effect of allatostatin in $\mathrm{JH}$ synthesis remains inconclusive because of the constant low quantity of $\mathrm{JHs}$ being detected in insects (Audsley et al., 1999). In normal JH regulation, degradation of
JHs is mediated by three enzymes: - juvenile hormone esterase (JHE), juvenile hormone epoxide hydrolase (JHEH), and juvenile hormone diol kinase (JHDK) (Yang et al., 2016). Yang et al. (2016) reported gene expressions of BdJHEH2 and BdJHDK in Bacterocera dorsalis (Diptera: Tephritidae) during larval-pupal transition and the genes suggested that $\mathrm{JH}$ was degraded in the preparation to pupate because low JH was required for successful larval-pupal development (Yang et al., 2016). Requirement of low JH during larval-larval moulting from early to late third instar larva followed by high demand $\mathrm{JH}$ degradation, which high expression of BdJHEH3 will be needed for this moulting to be successfully occurred. Adults of 7-days-old and 10- days-old B. dorsalis exhibited high expression of these three genes indicating that $\mathrm{JH}$ degradation was required for sexual maturation (Yang et al., 2016). In summary, the abundance of these genes found in the fat body and Malpighian tubules of $B$. dorsalis suggested that excretion of degraded $\mathrm{JH}$ was part of $\mathrm{JH}$ regulation.

Juvenile hormone biosynthetic pathway

Juvenile hormones are identified based on their chain length of 16-19 carbon atoms (Gullan \& Cranston, 2010). JH is synthesized in CA through the mevalonate pathway (MVAP) in the early stage and undergo $\mathrm{JH}$ branching in the later stage of biosynthetic pathway. This pathway was reviewed by Noriega (2014) where the synthesis of JH in CA comprises of 13 enzymatic reactions which involves eight enzymes in early MVAP and five enzymes in the JH branch (Noriega, 2014; Huang et al., 2015). The early step of MVAP includes the conversion of Acetyl-CoA to farnesyl pyrophosphate (FPP). Next, oxidation of FPP to farnesoic acid (FA) occurred and later transformed into a final product namely $\mathrm{JH}$. The last step in the JH branching was mediated by two enzymes genes, juvenile hormone acid O-methyltransferase (JHAMT) and methyl farnesoate epoxidase (CYP 15A1) (Marchal et al., 2011). JHAMT catalysed the FA and JH acid to the methyl ester forms which are $\mathrm{JH}$ and MF. Epoxidation of MF and $\mathrm{JH}$ is encoded by P450 enzyme with the expression of CYP 15A1. However, the late stage of MVAP is dependent on the order of an insects (Marchal et al., 2011). The late stage can precede with epoxidation followed by methylation in Lepidoptera or vice versa in Diptera, Coleoptera, Orthroptera, or Dictyoptera (Huang et al., 2015). Details on the pathway of JH biosynthesis (Figure 1) was reviewed by Noriega (2014) and includes the complete JH pathway and branching. However, Di Wen (2015) reported that the $\mathrm{JH}$ synthesis pathway of Drosophila is more complicated compared to other insects because of the presence of JHB III. The common precursor of JH present in Drosophila is believed to be farnesoic acid rather than methyl farnesoate. Methyl farnesoate is also reported to have a dual role as a hormone and precursor of JHBIII in Drosophila. The juvenile hormone acid methyltransferase (JHAMT) is known to be involved in JHB III biosynthesis only but not in JH III (Di-Wen et al., 2015). However, the complete pathway of $\mathrm{JH}$ biosynthesis is still unknown. Previous studies (Richards et al., 1989; Bylemans et al.,1998; Shiga et al., 2003) revealed a possibility of different biosynthesis in the type of $\mathrm{JH}$ and among different orders of insect. In other words, JH $0, \mathrm{JH}$ I, and JH II may have different biosynthesis pathways that have yet to be explored.

\section{Juvenile hormone control and regulation in insects}

$\mathrm{JH}$ has been identified to control and regulate various functions in insects from all order of insects. In this section, the control and regulation will be discussed among the different insect orders. The control of reproduction and development seem to be the most important one, as it the 


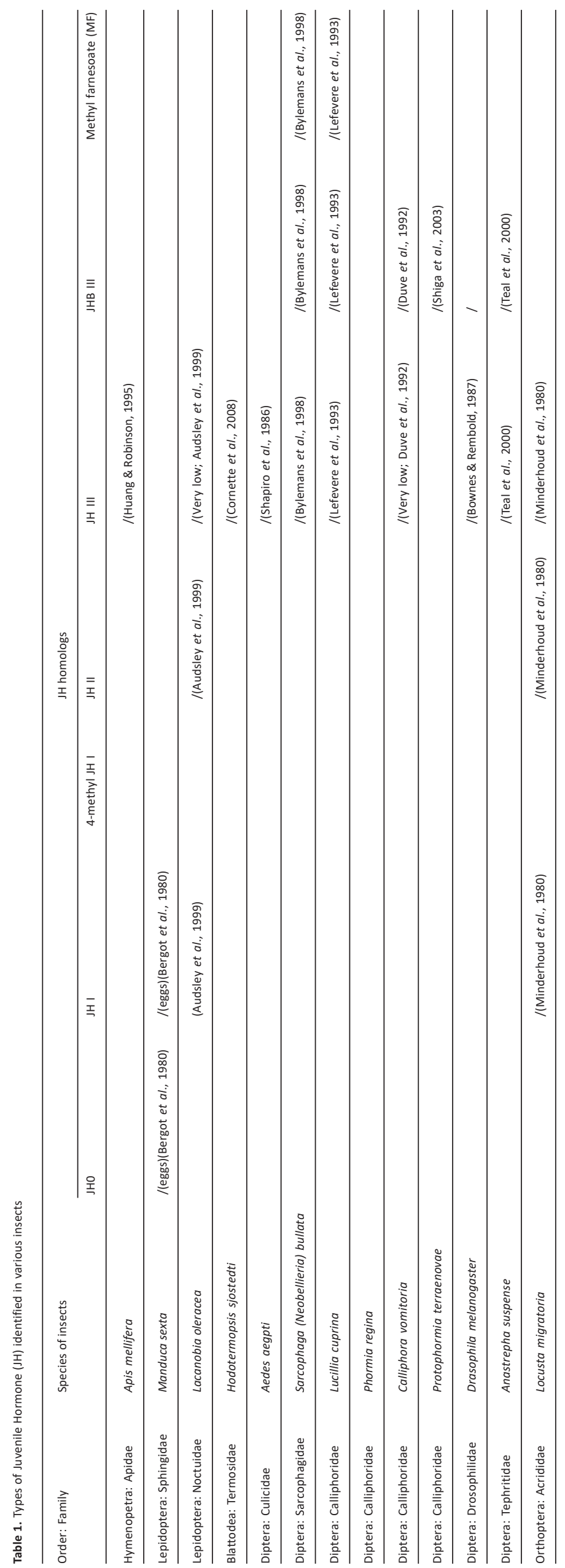




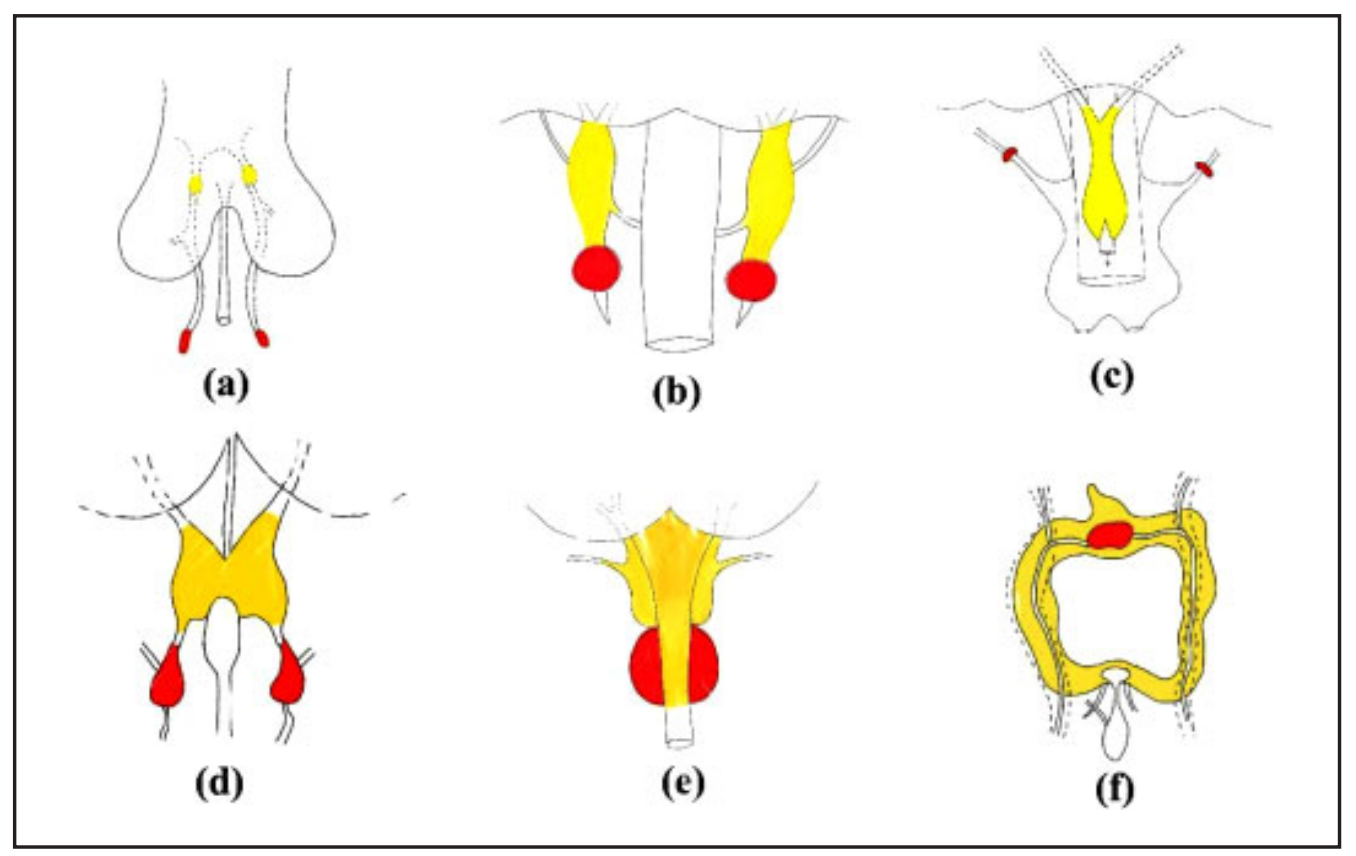

Figure 1. Morphological position of the retrocerebral glands in insects. (a) Lateralized types, (b) distal lateralized type, (c) ventral type, (d) semicentralized type, (e) centralized type, and (f) annular type. Red area represented corpora allata and yellow area represent corpora cardiaca. Adapted from Cassier (1979).

actively being studied in the past few years involving various insect species.

Despite the various forms of JH homologs, they shared the same control and regulations. In Diptera, three homologs were present in the extract of Musca domestica (Muscidae) and Sarcophaga bullata (Sarcophagidae) namely JH I, JH II, and JH III (Lefevere et al., 1993). However, further studies disputed the presence of $\mathrm{JH}$ II in M. domestica and S. bullata (Trumann, 1974; Girard et al., 1976; Schooley et al., 1976). Following this, a confirmation was made that $\mathrm{JH}$ III is the only principal $\mathrm{JH}$ found in cyclorrhapha Diptera. This JH is found in Drosophila hydei, D. melanogaster, Calliphora vomitoria (Calliphoridae), and Phormia regina (Calliphoridae) (Bownes \& Rembold, 1987; Duve et al., 1992)

In reproduction, not only JHs control the vitellogenesis and oogenesis but also regulate the reproductive behaviour and pheromone production in adult insects (Raushenbach et al., 2004). JH titre is reported to rise immediately after the emergence of both male and female $D$. melanogaster: $-6.9 \mathrm{pmol} / \mathrm{g}$ of wet weight in females and $5.3 \mathrm{pmol} / \mathrm{g}$ wet weight in males (Nijhout \& Williams, 1974). The increased concentration of $\mathrm{JH}$ in haemolymph immediately after emergence involves an initiation of the transcription and translation machinery in female's fat body for the induction of the first oogenesis cycle (reviewed by Wyatt \& Davey, 1996). This result is consistent with other studies where there was high titre of $\mathrm{JH}$ in the females of $A$. aegypti (Diptera: Culicidae) and both females and males of Neobellieria (Sarcophaga) bullata (Diptera: Sarcophagidae) immediately after eclosion but rapidly declined thereafter. Teal et al. (2000) proposed that the increase of JH titre five days after eclosion in males $N$. bullata and $D$. melanogaster were associated with reproductive maturity and sexual signalling. However, females have lower titres compared to the males of the same age and these differences were related to the presence of an allostatic-like compound found in the ovaries of both $D$. melanogaster and N. bullata females (Bownes \& Rembold, 1987; Bylemans et al., 1998). This allostatic-like compound may inhibit the biosynthesis of JH III and JHB III present in both insects which explained the rapid decline of these hormone titres in females. In males, the titre increases with mating behaviour as reported in Anastrepha suspense (Diptera: Tephritidae), where a 3 -fold increase of $\mathrm{JH}$ concentration were observed in mated young males compared to the virgin males (Teal et al., 2000).

$\mathrm{JH}$ also plays a part in the production of eggs, where a high concentration of $\mathrm{JH}$ is correlated with high productions of eggs and a low concentration of $\mathrm{JH}$ is accompanied by low eggs production (Yamamoto et al., 2013). Low JH production is due to the knockout of CA (CAKO) in females of D. melanogaster, which is the site of $\mathrm{JH}$ production and synthesis. Even though the correlation existed between $\mathrm{JH}$ and egg production, Yamamoto et al. found that some CAKO females produced a similar quantity of eggs in comparison to wild strain flies. This situation may be due to sufficient amounts of $\mathrm{JH}$ being produced before the knockout and hence the egg production is similar to the wild strains. As mentioned previously, the brain may play a part in sending the signal to the other accessory gland to increase $\mathrm{JH}$ synthesis before the CA is shutting down. In addition, JH was observed to be involved in oogenesis when a sharp decline of $\mathrm{JH}$ titres in $A$. aegypti were observed immediately after the blood meal and the rise of JH took place between 36 to 48 hours post-feeding (Shapiro et al., 1986). The decline of JH in early blood meals after eclosion create an opportunity for 20-hydroecdysone $(20 E)$ to release a neurosecretory hormone for egg development (Shapiro et al., 1986). A decline of JH level may be due to the action of juvenile hormone esterase, excretion, or the activities of its CA (Shapiro et al., 1986). The second blood meal in mosquitoes trigger the development of second follicles which will cause the rise of $\mathrm{JH}$ and a new cycle of egg development. In flesh fly, N. bullata, the increase of JH titres was more rapid when compared to $A$. aegypti which took an additional one day in both males and females following a liver and blood meal (Bylemans et al., 1998). However, the decline of $\mathrm{JH}$ in $\mathrm{N}$. bullata females a few days 


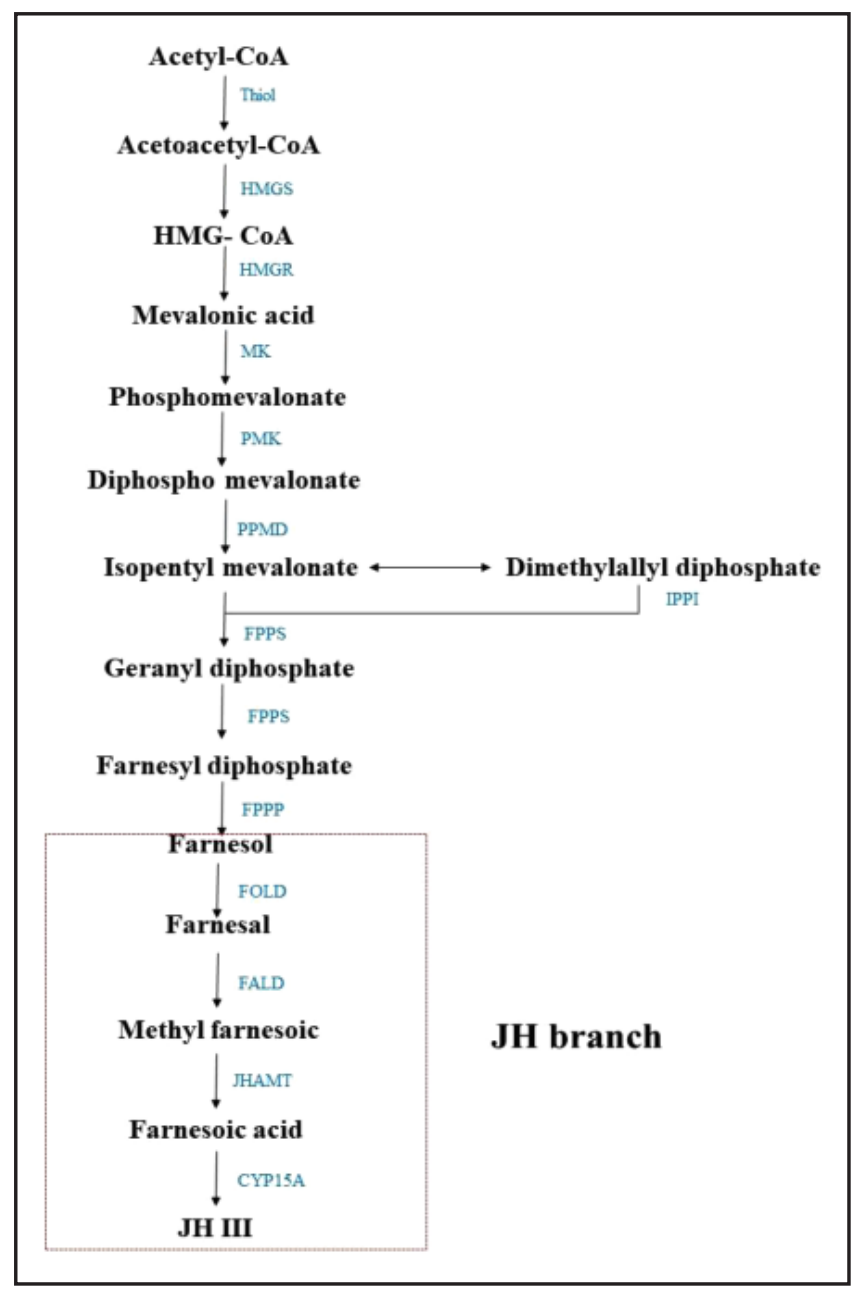

Figure 2. JH III biosynthesis pathway adapted from Noriega (2014). JH branch in the biosynthesis is in the red box. The enzymes involved are in blue. Abbreviations: Thiol= Acetoacetyl- CoA thiolase; HMGS= HMGCoA synthase; $\mathrm{HMGR}=\mathrm{HMG}-\mathrm{Co}$ A reductase; $\mathrm{MK}=$ Mevalonate kinase; PMK= Phosphomevalonate kinase; $P P M D=$ Diphosphomevalonate kinase; || $\mathrm{PI}=\mid$ sophentenyl diphosphate isomerase; FPPS= Farnesyl diphosphate isomerase synthase; FPPP= Farnesyl diphosphate synthase; $F O L D=$ Farnesol dehydrogenase; $F A L D=$ Farnesal dehydrogenase; JHAMT= Juvenile hormone acid methyl transferase; CYP15A= Methyl farnesoate epoxidase.

after the liver meal showed a similar trend with A. aegypti, demonstrating the involvement of $\mathrm{JH}$ in vitellogenesis (Bylemans et al., 1998).

In D. melanogaster, fluctuating patterns of $\mathrm{JH}$ titres were demonstrated throughout larval development until the prepupal stage (Bownes \& Rembold, 1987). First and second instar of $D$. melanogaster larvae showed high titres of JH (1.3 $\mathrm{pmol} / \mathrm{g}$ fresh weight) (Bownes \& Rembold, 1987). However, third instar and prepupa showed low titre and pupae did not show any detectable titre of JH. Reappearance of $\mathrm{JH}$ in the prepupal stage is required to prevent precocious metamorphosis in some tissues such as the eyes, the optic lobe, and the ventral diaphragm (Riddiford, 2012). Larvalpupal development was interrupted when the secretion of prothoracicotrophic hormone (PTTH) was inhibited by the brain in the presence of high JH concentration (Riddiford, 2012). As such, elimination of JH in haemolymph was required for the secretion of PTTH from the brain (Nijhout \& Williams, 1974; Riddiford, 2012).

$\mathrm{JH}$ regulation in moulting is further described at the molecular level when high Krüppel-homolog $1(K r-h 1)$ gene expression is required in larval-larval moulting of Tribolium castaneum (Coleoptera: Tenebrionidae) and Drosophila (Riddiford, 2012; Jindra, 2019). Expression of $K r$-h1 was high in both Tribolium and Drosophila throughout larval stages. Cessation of feeding and the wandering phase were triggered by the low gene expression which subsequently led to larvalpupal moulting (Riddiford, 2012). Suppression of $K r-h 1$ is regulated by histone deacetylase 1(HDAC 1) (George et al., 2019) with maximum mRNA expression of HDAC 1 was detected after 24 hours of entering the pupal stage. George et al. (2019) suggested that expression of $K r-h 1$ was related to $\mathrm{JH}$ concentration. In burying beetles, Nicropharus orbicollis (Coleoptera: Silphidae), JH did not seem to be involved in ovarian maturation but involved in behavioural regulation instead (Trumbo, 1994). The spiking of JH titres was detected in Ni. orbicollis upon the discovery of rat carcasses. The peak value of JH upon the discovery of carcasses were accompanied by four behaviour changes including palpating, lifting, walking around carcasses, and making forays into surrounding soils (Trumbo et al., 1994). However, the action of $\mathrm{JH}$ in the behavioural regulation is still not fully understood.

In social insects, JH has a different role compared to non-social insects. $\mathrm{JH}$ has been known to be involved in the polyethism (i.e., age polyethism and caste polyethism) and also behavioural changes. Polyethism is defined as functional specialization in different members of a colony of social insects, which lead to a division of labour (i.e., organisation on insect society that consists of different activities performed simultaneously by a specialized group of individuals). Polyethism can be categorized into age polyethism and caste polyethism. For example, honey bees, Apis mellifera (Hymenoptera: Apidae) are one of the social insects that undergo age polyethism, which is related to changes of division of labour as they age (Huang \& Robinson, 1995). For instance, the foragers have high JH titre compared to young Ap. mellifera. Huang and Robison (1995) also reported that the titres of $\mathrm{JH}$ are directly proportional with the age of honey bees, although low titre of $\mathrm{JH}$ was reported in inactive foragers and young Ap. mellifera. However, foraging activity was not totally dependent on the high titre of $\mathrm{JH}$ as active foragers in cold weather had a low titre than the active forager in warm weather. When compared with the young bees (which are non-foragers), the foraging bees in the cold weather exhibited a higher $\mathrm{JH}$. This showed that $\mathrm{JH}$ titre increases directly with the age of honey bees and also environmental temperature. Note that $\mathrm{JH}$ response towards temperature alone will not affect honey bees division of labour (Huang \& Robinson, 1995).

In addition, JH is playing a part in the pre-vitellogenic events of termites, Hodotermopsis sjostedti (Blattodea: Termosidae), Reticulitermes speratus (Blattodea: Rhinotermitidae), and Cryptotermes secundus (Blattodea: Kalotermitidae). High titre of JH during pre-vitellogenic events activates the vitellogenin synthesis (Cornette et al., 2008; Santos et al., 2018). The relationship between JH and polyethism was demonstrated in caste determination of damp wood termites, $H$. sjostedti. During moulting, high $\mathrm{JH}$ titre was associated with pre-soldier moulting while the moulting of nymph to the alate form showed low JH titres (Cornette et al., 2008). However, the titres started to fluctuate when soldiers reach maturity, in spite of these changes, titres in soldier termites were still higher than pseudergates termites (worker caste termites that unable to become a winged imago termites) (Cornette et al., 2008). This high titre may be linked to an increased volume of corpora allata in the soldiers. Yet, moulting of nymphs into pseudergates instead of alates due to the increase of JH titres may be due 
to the aggressive conspecific interaction in termites (Cornette et al., 2008). In a recent study by Oguchi et al. (2020), the differentiation of pseudergates to neotenic forms was depending on the presence of neotenics in the colony and this was regulated by the concentration of JH. Juvenile hormone was the key player in controlling the caste differentiation during the inter-molt of termites which will later determine the fates of the caste. Based on the experiment, the presence of neotenics in the colony will accelerate the differentiation of the opposite sex pseudergates to neotenics. However, the presence of male neotenics were able to maintain a low level of $\mathrm{JH}$ that led to the moulting of pseudergates to neotenics. In contrast, the presence of female neotenics, have showed high level of $\mathrm{JH}$ in the first three days, and then a gradual decrease, eventually reached the lowest level of JH on day seven. This differentiation may be caused by the urgency of a male to copulate due to the intraspecific competition (Oguchi et al., 2020).

In a recent study, behavioural roles of $\mathrm{JH}$ in honey bee larvae were studied by exposure to juvenile hormone analogue (JHA), pyriproxyfen, which subsequently showed an effect on behavioural change in the resulting adults (Fourrier, 2015). The pyriproxyfen-exposed adults did not perform many social tasks (i.e., brood care and ventilation) but rather performed more non-social tasks (i.e., selfgrooming, inactive, and walking instead of flying). Exposure of high JHA concentration may affect brain development and cognitive abilities of the honey bee and subsequently alter social performance that eventually led to its rejection by nestmates (Fourrier, 2015).

\section{Factors affecting $\mathrm{JH}$ activities}

As previously mentioned, JHs regulate many physiological activities in insects such as development, reproduction, polyethism, and behaviour. JHs are known to be declining in older larvae until reaching adulthood under normal regulation (Bownes \& Rembold, 1987). However, some factors may cause interruptions in the regulation and lead to the changes in physiological activities. Common effects such as prolong phagoperiod in larvae and delayed pupation occur due to rising JH titres in the haemolymph (Slama \& Williams, 1965; Saunders et al., 1990). Albeit low titres of JHs can also be a consequence due to environmental stress faced by insects.

One of the factors that affect $\mathrm{JH}$ activities is plant extracts, specifically balsam fir (Abies balsamea) that can be found in most American newspapers and some paper towels. This discovery was reported by Slama and Williams in 1965, when linden bug (Pyrrhocoris apterus) showed high titre of JH after being exposed to the plant extract. Following the high titre of $\mathrm{JH}$, larvae failed to moult into adults and most of the larvae died without reaching adulthood. Slama and Williams (1965) also reported that CA was not involved in the sensitivity of $P$. apterus towards the plant extract. Involvement of CA was not observed because the plant extract was applied directly to the larvae rather than injecting it into the haemolymph. Application of the extract to the other species of Hemiptera such as Oncopeltus fasciatus and Rhodnius prolixus did not show the same effect as in P. apterus. This indicates that the balsam fir extract cause changes of $\mathrm{JH}$ concentration in certain insect species only. This suggests that the plant extract acts as $\mathrm{JH}$ agonists (JHAs) to stimulate $\mathrm{JH}$ receptors.

Nevertheless, plant compounds can also act as JH antagonists (JHANs) that inhibit the binding of $\mathrm{JH}$ to its receptor, known as Methoprene-tolerant (Met). Diterpene from two plant extracts, the fruits of Lindera erythrocarpa and the roots of Solidago serotine, showed relatively strong and mild activity in the regulation of $\mathrm{JH}$ in $A$. aegypti, respectively. Both diterpene LE3B (Kanakugiol) and SS5A (Kingidiol) caused mortality in third instars, disrupted ovarian follicles development, and also cause ovarian retardation in females of $A$. aegypti. Retardation of $A$. aegypti female ovaries may be caused by precocious development by LE3B applied on the second instar during metamorphosis (Lee et al., 2015). Another factor that affects the titres of $\mathrm{JH}$ is seasonal changes. Photoperiod and temperature changes in each season effect the titres of JH in many insects. Saunders et al. (1990) reported that short-day photoperiod affected JH biosynthesis in $D$. melanogaster. Ovarian diapause was induced in short-day photoperiod that was regulated by $\mathrm{JH}$ titres in the haemolymph. The relation of $\mathrm{JH}$ titres in the haemolymph and photoperiod was measured by using the uptake of yolk proteins (YPs) by the ovaries of female $D$. melanogaster. The uptakes of YPs were low in a short-day photoperiod. This is because the synthesis of $\mathrm{JH}$ may not occur after a certain period of diapause (Saunders et al., 1990). The YPs tend to accumulate in the haemolymph and cannot be taken up by the ovaries (Saunders et al., 1990). Vitellogenesis is not complete in this situation. Vitellogenin synthesis is resumed in $D$. melanogaster by transferring the colony into a higher temperature $\left(25^{\circ} \mathrm{C}\right)$ and 12:12 light:dark photoperiod in order to break the ovarian diapause. However, the colony that were reared in short-day photoperiod for a long period (i.e., a month) did not recover from ovarian diapause following the method stated above. Another method that was applied to break the diapause was the topical application of $\mathrm{JH}$ (Saunders et al., 1990). Increasing JH in the haemolymph will increase the uptake of YPs by the ovaries. The correlation of $\mathrm{JH}$ and ovarian diapause was observed in non-diapausing flies that produced four times more JH than the diapausing flies (Saunders et al., 1990). The flies that produced more JH in the haemolymph were likely to undergo vitellogenesis (Saunders et al., 1990). Diapause-induced Pr. terraenovae also showed a low $\mathrm{JH}$ synthesis similar to diapause-induced $D$. melanogaster (Shiga et al., 2003).

It is known that JH titres are correlated with temperature. To further understand the effect of temperature on $\mathrm{JH}$ titres, foraging bees with high $\mathrm{JH}$ titres were placed into a cold room during summer (Huang et al., 1995). Eight days after the placement, A. mellifera showed a decline in the JH titres. Damp wood termites, Hodotermopsis sjostedti, also displayed temperature effects on $\mathrm{JH}$ but it is only affecting a certain caste which is the pseudergates termites (Cornette et al., 2008). Pseudergates termites experienced a dropped of $\mathrm{JH}$ titres during winter but the $\mathrm{JH}$ titres in soldier termites were similar to the warm weather despite the drop in temperature (Cornette et al., 2008).

Diet may also affect the production of $\mathrm{JH}$. Duve et al. (1992) reported that unrestricted diet in blow flies, C. vomitoria showed high production of $\mathrm{JH}$ and oocytes development in the first gonadotrophic cycle. In the laboratory, colonies where a restricted diet (i.e., sugar, water, meat) was given, the $\mathrm{JH}$ produced was less than $50 \%$. Bylemans et al. (1998) reported that the diet only affects biosynthesis of $\mathrm{JH}$ in female flesh flies, $N$. bullata, and not the males. This is based on an immediate increase of $\mathrm{JH}$ after a liver meal compared to males that showed a slower increase (Bylemans et al., 2015).

Starvation of fruit flies, $B$. dorsalis, demonstrated that the low expression of BdJHEH gene was responsible for the degradation of $\mathrm{JH}$. Starvation can cause low degradation of $\mathrm{JH}$ in their haemolymph which contradicted with previous studies by Duve et al. (1992). Thus, high JH in the haemolymph causes slower larval-larval moult. However, BdJHDK gene 
was highly expressed during insect starvation and caused precocious metamorphosis. In the adult females of Drosophila virilis (Diptera: Drosophilidae), starvation caused low production of eggs and a delay in oviposition (Raushenbach et al., 2004). This is caused by the low degeneration of $\mathrm{JH}$ which affect the production of 20-hydroxyecdysone (20E) that is important for the late stages of oogenesis.

\section{Detection and quantification of juvenile hormone}

Identification and quantification of $\mathrm{JH}$ had been performed in various method, ranging from classical method, bioassay, or physicochemical assay. In the early research, parabiosis and CA implantation was employed to study the $\mathrm{JH}$ synthesis (Villalobos-Sambucaro et al., 2020). Radiochemical assays, radioimmunoassay, and physicochemical methods which include gas chromatography (GC), liquid chromatography (LC), and spectrophotometry are the methods that being applied in $\mathrm{JH}$ studies to identify and quantify the hormone.

Radiochemical assay (RCA) is one of the common methods to analyse the rate of biosynthesis of $\mathrm{JH}$ in corpora allata. This assay is performed by tagging the compound with a radioactive isotope and incubation of the tissue (Bylemans et al., 1998). Observations were made in RCA by the incubation of brain-corpora cardiaca-corpora allata $(\mathrm{Br}$ CC-CA) and corpora cardiaca-copora allata (CC-CA). The rate of biosynthesis between these two complexes was then compared and the result showed higher biosynthesis in $\mathrm{Br}$ CC-CA than the CC-CA incubated without the brain. The synthesis is proven by the implantation of an active CA from young larvae into older larvae of $M$. sexta with less active CA. This implantation resulted in an increase of $\mathrm{JH}$ level in the haemolymph (Huibregtse-Minderhoud et al., 1980). In vitro incubation of CA in radiochemical assay (RCA) also showed $\mathrm{JH}$ synthesis by the CA. A study on locusts, Locusta migratoria by Huibregtse-Minderhoud et al. (1980) used capillary GC to detect three $\mathrm{JH}$ homologs; JH I, JH II, and JH III. A concentration of $2.3 \mathrm{ng} / \mathrm{g}$ haemolymph of $\mathrm{JH}$ III was detected in 0-24 hours fifth instar larvae compared to 24-48 hours larvae at minimum of $0.9 \mathrm{ng} / \mathrm{g}$ haemolymph which is much lower compared to the young larvae. After 18 days, upon reaching adult stage, the reading of $\mathrm{JH}$ III concentration is higher with 36.0-74 ng/g haemolymph. Whereas, JH I and JH II have low concentrations, less than $0.3-2.5 \mathrm{ng} / \mathrm{g}$ haemolymph, and cannot be detected in some samples.

In 1981, Bergot et al. developed a rapid, specific, and sensitive method for the extraction, purification, and quantitative determination of four known $\mathrm{JHs}$ which are $\mathrm{JH}$ $0, \mathrm{JH} \mathrm{I}, \mathrm{JH} \mathrm{II}$, and JH III. This method involved the application of Gas Chromatography Mass-Spectrometry (GC-MS) in two lepidopteran species; $M$. sexta and Heliothis virescens. The method developed by Bergot et al. has simplified and shortened the purification process of the sample and only a small sample size is required for the analysis. Furthermore, higher sensitivities can be achieved using this method. Adapting the method, Tobe et al. (1985) detected 46-740 $\mathrm{ng} / \mathrm{g}$ of JH III in the whole-body extract of Diploptera punctata for each developmental stage ( $n=12-15$ individuals).

In the recent advancement of technology, simultaneous identification and quantification of $\mathrm{JH}$ can be performed in a single run in LC Ramirez et al. (2020). This method allowed the identification and quantification of $\mathrm{JH} \mathrm{I}, \mathrm{JH} \mathrm{II}, \mathrm{JH} \mathrm{III}, \mathrm{JHB}$ III, and Juvenile Hormone Skipped-Bisepoxide (JHSB III). Employment of LC coupled to electrospray tandem mass spectrometry (LC-MS/MS) permitted the simultaneous identification and quantification based on $\mathrm{JH}$ structural features. This developed protocol could detect a femtomole range of the $\mathrm{JH}$ concentrations, allowing analysis of an individual insect. Previous methods developed by RiveraPerez et al. (2012) are also able to detect a femtomole range of $\mathrm{JH}$ titres but this result is obtained by tagging the $\mathrm{JH}$ to the fluorescent tag prior to the High-Performance Liquid Chromatography (HPLC). This procedure can be applied to three type of samples which are the haemolymph, whole tissue, and CA-CC samples.

\section{Application of $\mathrm{JH}$ in vector control and its future applications}

The application of $\mathrm{JH}$ is mainly centred on the agricultural and vector control industries especially on the development of insecticides or insect growth regulators (IGRs). The use of pesticides renders significant negative impacts to human and environmental health. IGRs do not deliver a rapid termination on the insect lives but it affects the biology of insects with long term effects. In general, JH manipulation in insect pest and vector control was executed in the form of $\mathrm{JH}$ mimicry or $\mathrm{JH}$ analogues. These analogues include methoprene, pyriproxyfen, and hydroprene. The detailed management and potential use of IGRs was reviewed by Mondal and Parween (2001).

Despite of its effectiveness, tolerance and resistance towards IGRs by some insects such as mosquitoes, house flies, and flour beetles were reported (Cornel et al., 2002). Resistance towards methoprene maybe caused by (i) reduced penetration of the substance into the system; (ii) increased excretions, and (iii) insensitivity of the target site. While, in $D$. melanogaster, the resistance was caused by mutagenesis of the Met genes which is the receptor for JH (Minkoff \& Wilson, 1992). In a review by Parthasarathy and Palli (2021), the tolerance of the JHA is simply depended on the specific gene in the targeted insects. A study on Tribolium castaneum (Coleoptera: Tenebrionidae) showed three different outcomes from the same treatment: - (i) failure of $T$. castaneum to emerge as adult; (ii) successfully emerged as adult but produced no offspring, and (iii) emerged as adult and produced normal offspring (Wijayaratne et al., 2012). Following these outcomes, males $T$. castaneum were more susceptible to the treatment compared to females. Treatment applied to females $T$. castaneum did not show significant differences in the production of normal offspring compared to untreated females. In contrast, untreated females mated with treated males showed differences in the production of eggs (e.g., failure to hatch). This indicated that males were more susceptible than females against treatment. Susceptibility of males may be caused by the disruption in the process of spermatogenesis by methoprene during the developmental cycle (Wijayaratne et al., 2012). Thus, disruption in reproductive development in insects reduces the progeny of insect population to achieve the purpose of pest control (Shapiro et al., 1986; Wijayaratne et al., 2012).

One of the most potent $\mathrm{JH}$ analogues used in insect population control is pyriproxyfen or 2 [I-methyl-2-(4 phenoxypheoxy) ethoxy] pyridine (Ishaaya \& Horowitz, 1992). They reported the effect of pyriproxyfen on the development of sweet potato white flies, Bemisia tabaci (Hemiptera: Aleyrididae) which is known to infest cotton, vegetables, and ornamental plants. Pyriproxyfen did show a positive response in the suppression of the development of $B$. tabaci in every developmental stage. Inviable eggs were produced from adult females treated with the substance. The direct effect of pyriproxyfen to eggs was only seen in the 0-1 dayold eggs and not on the older eggs. The same was observed in the larval stages. However, the effects were only seen in the later stages when the pupae of the treated larvae failed to emerge as adults. Pyriproxyfen also showed localized effect on the area of interest, where eggs laid on specific 
targeted area of the leaves failed to hatch (Ishaaya \& Horowitz, 1992).

$\mathrm{JH}$ analogues (JHAs) has been investigated in insect vectors such as mosquitoes and triatomid bugs. Mosquito is a known vector of various tropical diseases such as dengue fever, malaria, and Zika. While triatomes are vectors of Chagas disease and both of these insects required a blood meat for oocytes productions (Ramos et al., 2020). Associations of these insects with human is concerning as they will spread diseases among human populations and therefore vector control programs are paramount important in this context. Methoprene, also known as isopropyl (2E, 4E)-11-methoxy-3,7,11-trimethyl-2-4-dodecadienoate, is the most common JH analogue used to control mosquitoes and fruit flies (Wilson \& Turner, 1992). Shapiro et al. (1986) reported a five time increase in the amount of $\mathrm{JH}$ in unfed A. aegypti females when methoprene was applied topically $(100 \mathrm{pg})$. The application of JHA causes vitellogenesis to occur in female $A$. aegypti and most of the females successfully oviposited. However, most of the eggs produced were not viable, which suggested that high levels of $\mathrm{JH}$ caused failure in embryogenesis (Shapiro et al., 1986).

JHA is also known to cause defect and death in mosquito larvae (Parthasarathy \& Palli, 2021). It may produce a larvapupal intermediate during the larval-pupal critical period. Continuous exposure to $\mathrm{JH}$ after the larval-pupal critical period will cause mortality during the prepupal stage (Braga et al., 2005). Methoprene has been reported to control A. aegypti successfully (Braga et al., 2005a, 2005b). While pyriproxyfen is able to inhibit adult emergence of $A$. vigilax and A. japonicus (Webb et al., 2012; Tuten et al., 2016). Furthermore, methoprene is able to control horn flies and house flies (Beadles et al., 1975; Miller et al., 1977; Breeden et al., 1980). However, it is interesting to note that no JH homologs was found in ticks (Acari). The JHA that has been studied thus far in insect vector is summarised in Table 2.

In a recent study, Chang et al. (2021) shows that the increase of $\mathrm{JH}$ in an adult $A$. aegypti reduces their immunity and that they are more susceptible to an infection. This study revealed that the disruption of $\mathrm{JH}$ signalling not only affect reproduction but also the insect immunity. In triatomid bugs, there is no reports on the JHA used to control this insect vector as the $\mathrm{JH}$ that is specific to this organism was just discovered recently (Villalobos-Sambucaro et al., 2020). The $\mathrm{JH}$ discovered is thoroughly studies for a development of specific targeted JHA (Villalobos-Sambucaro et al., 2020). Some JHAs do showed high specificity to a specific insect order, one of the JHAs acting this way is methyl ester of 3,7,11-trimethyl-7,11-dichloro-2-dodecenicacid or "dihydrochloride" of methyl farnesoate (Masner et al., 1968). This substance showed potency in sterilization of the linden bug, Pyrrhocoris apterus. Female P. apterus showed high sensitivity to this substance as low as $0.1 \mu \mathrm{g}$ which is sufficient to cause partial viability of the eggs produced (Masner et al., 1968). However, no effect was observed in the developmental of first and fourth instars of $P$. apterus. This indicated that the effect was taking place during the embryogenesis. Yet, in some other species such as Lepidoptera, Coleoptera, Diptera, and some Heteroptera, it did not show any effect, suggesting high specificity of this substance (Masner et al., 1968).

Juvenile hormone chemistry, biosynthesis, and pathway have been extensively study for better understanding on its regulations in insects for a few decades. However, most of the studies were centred on $\mathrm{JH}$ application in the insect pest and vector control industry in the form of IGRs. JH analog are the most commonly used to regulate the $\mathrm{JH}$ titres in pest insects. High JH during embryogenesis caused non-viable egg production (Shapiro et al., 1986) which is efficient in controlling the insect pest and vector population. Altering the $\mathrm{JH}$ titre in larval stages is a useful strategy in vector population control because inducing $\mathrm{JH}$ titre in the old larvae stops adult transformation and resulted in the discontinuation of its life cycle (Bownes \& Rembold, 1987).

Declining trends of JHs during fly larval development may be useful in the determination of the age of the larvae. Larval age is used for the determination of minimum postmortem interval (mPMI) in forensic investigations (Goff, 1993), especially to differentiate the age of third instar and post feeding stages. It is known that the larvae of blow flies are common insect evidence found on decomposing remains (Lee et al., 2004). Furthermore, beetle larvae can also be used in the estimation of PMI at a later stage of body decomposition. We hypothesized that the levels of $\mathrm{JH}$ determined from fly larvae or beetle larvae during the discovery of a body can be used to determine PMI. Furthermore, the peak concentration of JH in carrion beetles during the discovery of the carcasses could potentially be useful in determining the time of the beetle arrival. Yet, to apply this knowledge in forensic entomology, extensive studies are required to fully understand the relationship of $\mathrm{JH}$ titres changes and the time of beetle arrival to the decomposing remains. Analysis of biological changes in each insect found at the crime scene can improve mPMI estimations. Although, a study focusing on the trends of JH in the development of a blowfly need to be performed to explore their application in forensic because of their value in $\mathrm{mPMI}$ estimation compared to beetles. Nevertheless, a baseline data on the $\mathrm{JH}$ changes in each insect species involved in crime scene investigation need to be constructed for its application in the real setting.

The other potential application of $\mathrm{JH}$ is in the recovery of an important social insects by studying evolution of $\mathrm{JH}$ in the regulation of insect behaviour. This may be advantageous in the recovery of a social insect colony. Instability of $\mathrm{JH}$ within the colony of a social insect may cause aggressiveness

Table 2. Application of JHA in controlling vector of diseases

\begin{tabular}{|c|c|c|c|}
\hline JHA & Vector & Life stages & Authors \\
\hline \multirow[t]{2}{*}{ Methoprene } & Ochlerotatus nigromaculis (Diptera: Culicidae) & Larvae & Cornel et al., 2002 \\
\hline & Aedes aegypti (Diptera: Culicidae) & Larvae & $\begin{array}{l}\text { Braga et al., 2005a, 2005b; } \\
\text { Wu et al., } 2006\end{array}$ \\
\hline Pyriproxyfen & Aedes vigilax (Diptera: Culicidae) & Larvae & Webb et al., 2012 \\
\hline Methoprene (added to the chicken feed) & Musca domestica (Diptera: Muscidae) & Adults & Breeden et al., 1980 \\
\hline Methoprene (added to water system) & Haematobia irritans (Diptera: Muscidae) & Adults & $\begin{array}{l}\text { Beadles et al., 1975; } \\
\text { Miller et al., } 1977 .\end{array}$ \\
\hline
\end{tabular}


and rejection behaviours by their nest mates which can lead to the collapse of a colony. These aggressive behaviours may be corrected by the application of JH. Acceptance rates by nestmates may be increases by manipulating the level of $\mathrm{JH}$ to induce social or non-social behaviour in social insects.

\section{CONCLUSION}

$\mathrm{JH}$ clearly have a complex and unique biosynthesis in each order of insects. Eight different $\mathrm{JH}$ homologs have been identified thus far and presented in a different combination for each insect order. These hormones are synthesized by the gland namely corpora allata (CA) which is situated near the brain.

JHs are known to regulate various physiological changes such as moulting, reproduction, polyethism, and behavioural changes. Due to the complexity of $\mathrm{JH}$ pathways and regulation, this has opened new research interests among researchers to fully understand the impact of $\mathrm{JHs}$ on every insect species. The relationship of JH titre which change with various abiotic factors such as temperature, photoperiod and also diet is an interesting area to be explored.

In addition, synthetic JH compounds have been widely applied in insect population control such as IGRs in the pest control industry. Due to its potency and environmental benefits compared to chemical pesticides, synthetic JH products have been widely used in the industry worldwide. Yet, a continuous effort should be conducted to understand the nature of each $\mathrm{JH}$ of every insect in molecular level for the production of effective vectors control compound. Nevertheless, the dynamics of JH titres in fly larval development on decomposing bodies might potentially be useful in the field of forensic entomology. As discussed in this review, extensive entomo-biochemistry studies are needed before the data can be applied in real forensic settings.

\section{ACKNOWLEDGEMENTS}

The first author was supported by the Fundamental Research Grant Scheme by the Ministry of Higher Education, Government of Malaysia [600-IRMI/FRGS 5/3 (103)].

\section{Conflict of Interest}

The author declares that there is no conflict of interest.

\section{REFERENCES}

Audsley, N., Weaver, R.J. \& Edwards, J.P. (1999). Juvenile hormone synthesis by corpora allata of tomato moth, Lacanobia oleracea (Lepodoptera: Noctuidae), and the effects of allatostatins and allatotrophin in vitro. European Journal of Entomology 96: 287-294.

Beadles, M.L., Miller, J.A., Chamberlain, W.F., Eschle, J.L. \& Harris, R.L. (1975). The horn fly: Methoprene in drinking water of cattle for control12. Journal of Economic Entomology 68: 781-785. https://doi.org/10.1093/jee/68.6.781

Bergot, B.J., Jamieson, G.C., Ratcliff, M.A. \& Schooley, D.A. (1980). JH zero: new naturally occurring insect juvenile hormone from developing embryos of the tobacco hornworm. Science 210: 336-338. https://doi.org/10.1126/ science.210.4467.336

Bergot, B.J., Ratcliff, M. \& Schooley, D.A. (1981). Method of the four juvenile hormones in insect tissue using gas chromatography-mass spectrometry. Journal of Chromatography 204: 231-244. https://doi.org/10.1016/S00219673(00)81664-7
Blight, M. \& Wenham, M.J. (1976). Identification of JH-III in haemolymph from adults and larvae of Schistocerca gregaria. Insect Biochemistry 6: 35-38. https://doi.org/ 10.1016/0020-1790(76)90056-1

Bownes, M. \& Rembold, H. (1987). The titre of juvenile hormone during the pupal and adult stages of the life cycle of Drosophila melanogaster. European Journal of Biochemistry 164: 709-712. https://doi.org/10.1111/j.14321033.1987.tb11184.x

Braga, I.A., Mello, C.B., Peixoto, A.A. \& Valle, D. (2005a). Evaluation pf methoprene effect on Aedes aegypti (Diptera: Culicidae) development in laboratory conditions. Memórias do Instituto Oswaldo Cruz 100: 435-440. https://doi.org/10.1590/s0074-02762005000400016

Braga, I.A., Mello, C.B., Montella, I.R., Lima, J.B., Martins, A.J., Madeiros, P.F. \& Valle, D. (2005b). Effectiveness ot methoprene, an insect growth regulator, against temephos-resistant Aedes aegypti populations from different Brazillian localities, under lanoratory conditions. Journal of Medical Entomology 42: 830-837.

Bylemans, D., Borovsky, D., Ujvary, I. \& Deloof, A. (1998). Biosynthesis and regulation of juvenile hormone III, juvenile hormone iii bisepoxide, and methyl farnesoate during the reproductive cycle of the grey fleshfly, Neobellieria (Sarcophaga) bullata. Archive of Insect Biochemistry 37: 248-256. https://doi.org/10.1002/(SICI)1520-6327(1998) 37:3\%3C248::AID-ARCH8\%3E3.0.CO;2-O

Cassier, P. (1979). The corpora allata of insects. International Review Cytology 57: 1-73. https://doi.org/10.1016/S00747696(08)61461-8

Chang, M.-M., Wang, Y.-H., Yang, Q.-T., Wang, X.-L., Wang, M., Raikhel, A.S. \& Zou, Z. (2021). Regulation of antimicrobial peptides by juvenile hormone and its receptor, Methoprene-tolerant, in mosquito Aedes aegypti. Insect Biochemistry and Molecular Biology 128: 103509. https:// doi.org/10.1016/j.ibmb.2020.103509

Cornel, A.J., Stanich, M.A., Mcabee, R.D. \& Mulligan, F.S. (2002). High level methoprene resistance in the mosquito Ochlerotatus nigromaculis (Ludlow) in Central California. \% Pest Management Science 58: 791-798. https://doi.org/ $10.1002 / p s .521$

Cornette, R., Gotoh, H., Koshikawa, S. \& Miura, T. (2008). Juvenile hormone titers and caste differentiation in the damp-wood termite Hodotermopsis sjostedti (Isoptera, Termopsidae). Journal of Insect Physiology 54: 922-930. https://doi.org/10.1016/j.jinsphys.2008.04.017

Di-Wen, Rivera-Perez, C., Abdou, M., Jia, Q., He, Q., Liu, X., Zyaan, O., Xu, J., Bendena, W.G. \& Tobe, S.S. (2015). Methyl farnesoate plays a dual role in regulating Drasophila metamorphosis. PLoS Genetics 11: e1005038. https:// doi.org/10.1371/journal.pgen.1005038

Duve, H., Thorpe, A., Yagi, K.J., Yu, C.G. \& Tobe, S.S. (1992). Factors affecting the biosynthesis and release of juvenile hormone bisepoxide in the adult blowfly Calliphora vomitoria. Journal of Insect Physiology 38: 575-585. https:// doi.org/10.1016/0022-1910(92)90109-Q

Emlen, D.J. \& Nijhout, H.F. (2001). Hormonal control of male horn length dimorphism in Orthophagus taurus (Coleoptera: Scarabaeidae): a second critical period of sensitivity to juvenile hormone. Journal of Insect Physiology 47: 1045-1054. https://doi.org/10.1016/S0022-1910(01)00084-1

Fourrier, J., Deschamps, M., Léa Droin, L., Alaux, C., Fortini, D., Beslay, D., Conte, Y.L., Devillers, J., Aupinel, P. \& Decourtye, A. (2015). Larval exposure to the juvenile hormone analog pyriproxyfen disrupts acceptance of and social behavior performance in adult honeybees. PLoS One 10: e0132985. https://doi.org/10.1371/journal.pone.0132985 
George, S., Gaddelapati, S.C. \& Reddy, S. (2019). Histone deacetylase 1 suppress Krüppel homolog 1 gene expression and influences juvenile hormone action of Tribolium castaneum. Proceedings of the National Academy of Science of the United State of America 116: 17759-17764. https://doi.org/10.1073/pnas.1909554116

Goff, M. (1993). Estimation of postmortem interval using arthropod development and successional patterns. Forensic Science Review 5: 82-94.

Gilbert, L.I. (2012). The Juvenile Hormone. In: Insect Endocrinology, W.G. Goodman \& M. Cusson (editors.) 1st Edition. London: Elsevier, pp. 310-365.

Gullan, P.J. \& Cranston, P.S. (2010). Internal Anatomy and Physiology. In: Insects: An Outline of Entomology, Gullan, P.J. \& Cranston, P.S. (editors) 4th Edition. United Kingdom: Wiley-Blackwell, pp. 63-67.

Hartfelder, K. (2000). Insect juvenile hormone: from "status quo" to high society. Brazilian Journal of Medical Biological Research 33: 157-177. https://doi.org/10.1590/S0100$879 \times 2000000200003$

Huang, J., Marchall, E., Hult, E.F. \& Tobe, S.S. (2015). Characterization of the juvenile hormone pathway in viviparous cockroach Diploptera punctata. PLoS One 10: e0117291. https://doi.org/10.1371/journal.pone.0117291

Huang, Z.Y. \& Robinson, G.E. (1995). Seasonal changes in juvenile hormone titers and rates of biosynthesis in honey bees. Journal of Comparative Physiology B 165: 18-28. https://doi.org/10.1007/BF00264682

Huibregtse-Minderhoud, L., Hondel-Fraken, M.A.M.V.V.D., Hoof, A.C.V.D.K.V., Biessels, H.W.A., Salemink, C.A., Horst, D.J.V.D. \& Beenakers, A.M.Th. (1980). Quantitative determination of juvenile hormones in the haemolymph of Locusta migratoria during normal development and after implantation of corpora allata. Journal of Insect Physiology 26: 627-631. https://doi.org/10.1016/0022-1910(80)90032-3

Ishaaya, I. \& Horowitz, A.R. (1992). Novel phenoxy juvenile hormone analog (pyriproxyfen) suppresses embryogenesis and adult emergence of sweet potato whitefly (Homoptera: Aleyrodidae). Journal of Economic Entomology 85: 2113-2117. https://doi.org/10.1093/jee/85.6.2113

Jindra, M. (2019). Where did the pupa come from? The timing of juvenile hormone signalling supports homology between stages of hemimetabolous and holometabolous insects. \%Philosophical Transactions of Royal Society of London Series B, Biological Science 374: 20190064. https:// doi.org/10.1098/rstb.2019.0064

Judy, K., Schooley, D., Hall, M.S., Bergot, B.J. \& Siddal, J.B. (1973). Chemical structure and absolute configuration of juvenile hormone from grasshopper corpora allata in vitro. Life Science 13: 1511-1516. https://doi.org/10.1016/0024-3205(73) 90139-2

Lee, H., Krishnasamy, M., Abdullah, A.G. \& Jeffery, J. (2004). Review of forensically important entomological specimens in the period of 1972-2002. Tropical Biomedicine 21: $69-75$

Lee, S.H., Oh, H.W., Fang, Y., An, S.B., Park, D.S., Song, H.H., Oh, S.R., Kim, S.Y., Kim, S. \& Kim, N. (2015). Identification of plant compounds that distrupt the insect juvenile hormone receptor complex. Proceedings of the National Academy of Science of the United State of America 112: 17331738. https://doi.org/10.1073/pnas.1424386112

Lefervere, K.S., Lacey, M.J., Smith, P.H. \& Roberts, B. (1993). Identification and quantification of juvenile hormone biosynthesis by larval and adult Australian sheep blowfly Lucillia cuprina (Diptera: Calliphoridae). Insect Biochemistry and Molecular Biology 23: 713-720. https://doi.org/10.1016/ 0965-1748(93)90045-T
Marchal, E., Zhang, J.R., Badisco, L., Verlinden, H., Hult, E.F., Van Wielendaele, P. \& Vanden Broeck, J. (2011). Final steps in juvenile hormone biosynthesis in the desert locust, Schistocerca gregaria. Insect Biochemistry and Molecular Biology 41: 219-227. https://doi.org/10.1016/j.ibmb.2010. 12.007

Masner, P., Sláma, K. \& Landa, V. (1968). Natural and synthetic materials with insect hormone activity: IV. Specific female sterility effects produced by a juvenile hormone analogue. Development 20: 25-31. https://doi.org/10.1242/ dev.20.1.25

Miller, J.A., Esehle, J.L., Hopkins, D.E., Wright, F.C. \& Matter, J.J. (1977). Methoprene for control of horn flies: A suppression program on the island of Molokai, Hawaii13. Journal of Economic Entomology 70: 417-423. https://doi.org/10.1093/ jee/70.4.417

Minkoff, C. \& Wilson, T. (1992). The competitive ability and fitness components of the methoprene tolerant (Met) Drasophila mutant resistant to juvenile hormone analogue insecticide. Genetics Society of America 131: 9197.

Mondal, K.A.M.S.H. \& Parween, S. (2001). Insects growth regulators and their potential in the management of stored-product insects pest. Intergrated Pest Management Reviews 5: 255-295. https://doi.org/10.1023/A:101290 1832162

Nijhout, H.F. \& Williams, C.M. (1974). Control of moulting and metamorphosis in the tobacco hornworm, Manduca Sexta (L.): cessation of juvenile hormone secretion as a trigger for pupation. Journal of Experimental Biology 61: 493-501. https://doi.org/10.1242/jeb.61.2.493

Noriega, F.G. (2014). Juvenile hormone biosynthesis in insects: what is new, what do we know, and what questions remains? International Scholarly Research Notices 2014: 967361. https://doi.org/10.1155/2014/967361

Oguchi, K., Sugime, Y., Shimoji, H., Hayashi, Y. \& Miura, T. (2020). Male neotenics reproductives accelerate additional differentiation of females reproductives by lowering $\mathrm{JH}$ titers in termites. Scientific Reports 10: 9345. https://doi.org/ 10.1038/s41598-020-66403-0

Ramirez, C.E., Nouzova, M., Michalkova, V., Fernandez-Lima, F. \& Noriega, F.G. (2020). Common structural features facilitate the simultaneous identification and quantification of the five most common juvenile hormones by liquid chromatography-tandem mass spectrometry. Insect Biochemistry and Molecular Biology 116: 103287. https:// doi.org/10.1016/j.ibmb.2019.103287

Rantala, M.J., Dubovskiy, I.M., PÖlkki, M., Krama, T., ContrerasGarduño, J. \& Krams, I.A. (2020). Effect of juvenile hormone on resistance against entomopathogenic fungi Metarhizium robertsii differs between sexes. Journal of Fungi 6: 298. https://doi.org/10.3390/jof6040298

Parthasarathy, R. \& Palli, S.R. (2021). Stage specific action of juvenile hormone analogs. Journal of Pesticide Science 46: 16-22. https://doi.org/10.1584/jpestics.D20-084

Ramos, F.B., Leyria, J., Nouzova, M., Fruterro, L.L., Noriega, F.G. \& Canasova, L.E. (2020). Juvenile hormone mediates lipid storage in the oocytes of Dipetalogaster maxima. Insect Biochemistry and Molecular Biology 133: 103499. https:// doi.org/10.1016/j.ibmb.2020.103499

Raushenbach, I.Y, Gruntenko, N.E., Bownes, M., Adonieva, N.V., Terashima, J., Karpova, E.K., Faddeeva, N.V. \& Chentsova, N.A. (2004). The role of juvenile hormone in the control of reproductive function in Drosophila virilis under nutritional stress. Insect Physiology 50: 323-330. https://doi.org/10.1016/ j.jinsphys.2004.02.001 
Richard, D.S., Applebaum, S.W., Sliter, T.J., Baker, F.C., Schooley, D.A., Reuter, C.C. \& Gilbert, L.I. (1989). Juvenile hormone bisepoxide biosynthesis in vitro by the ring gland of Drosophila melanogaster: a putative juvenile hormone in the higher Diptera. Proceedings of the National Academy of Science of the United State of America 86: 1421-1425. https:/ /doi.org/10.1073/pnas.86.4.1421

Riddiford, L.M. (2012). How does juvenile hormone control insect metamorphosis and reproduction. General and Comparative Endocrinology 179: 477-484. https://doi.org/ 10.1016/j.ygcen.2012.06.001

Rivera-Perez, C., Nouzova, M. \& Noriega, F.G. (2012). A quantitative assay for juvenile hormone and their precursors using fluorescent tags. PLoS One 7: e43784. https://doi.org/10.1371/journal.pone.0043784

Santos, C.G., Humann, F.C. \& Hartfelder, K. (2018). Juvenile hormone signalling in insect oogenesis. Current Opinion in Insect Science 31: 48-48. https://doi.org/10.1016/ j.cois.2018.07.010

Saunders, D.S., Richard, D.S., Applebaum, S.W., Ma, M. \& Gilbert, L.I. (1990). Photoperiodic diapause in Drosophila melanogaster involves a block to the juvenile hormone regulation of ovarian maturation. Genetic and Comparative Endocrinology 79: 174-184. https://doi.org/10.1016/00166480(90)90102-R

Shapiro, A.B., Wheelock, G.D., Hagedorn, H.H., Baker, F.C., Tsai, L.W. \& Schooley, D.A. (1986). Juvenile hormone and juvenile hormone esterase in adult females of the mosquito Aedes aegypti. Journal of Insect Physiology 32: 867-877. https:/ /doi.org/10.1016/0022-1910(86)90102-2

Shiga, S., Hamanaka, Y., Tatsu, Y., Okuda, T. \& Numata, H. (2003). Juvenile hormone biosynthesis in diapause and nondiapause females of adult blowfly Photophormia terraenovae. Zoological Science 20: 1199-1206. https://doi.org/ 10.2108/zsj.20.1199

Slama, K. \& Williams, C.M. (1965). Juvenile hormone activity for the bug Pyrrhocoris apterus. Proceedings of the National Academy of Science of the United State of America 54: 411414. https://dx.doi.org/10.1073\%2Fpnas.54.2.411

Teal, P.E.A. \& Gomez-Simutproveaux, A.T. (2000). Mating experience and juvenile hormone enhance sexual signalling and mating in male Caribbean fruit flies. Proceedings of the National Academy of Science of the United State of America 97: 3708-3712. https://doi.org/ 10.1073/pnas.97.7.3708

Tobe, S.S., Ruegg, R.P., Stay, B.A., Baker, F.C., Miller, C.A. \& Schooley, D.A. (1985). Juvenile hormone titre and regulation in cockroach Diploptera punctata. Experientia 41: 1028-1034. https://doi.org/10.1007/BF01952127

Trautmann, K.H., Masner, P., Schuler, A., Suchy, M. \& Wipf, H.K. (1974). Evidence of the juvenile hormone methyl (2E, 6E)10,11-epoxy-3,7,11-trimethyl-2,6-dodecadienoate $(\mathrm{JH}-3)$ in insects of four orders. Zeitschrift für Naturforschun 29c: 757759. https://doi.org/10.1515/znc-1974-11-1218
Trumbo, S.T., Borst, D.W. \& Robinson, G.E. (1994). Rapid elevation of juvenile hormone titer during behavioral assessment of the breeding resource by the burying beetle, Nicrophorus orbicollis. Journal of Insect Physiology 41: 535-543. https://doi.org/10.1016/0022-1910(94)00127-3

Van Den Hondel-Fraken, M.A.M., Van Den Broek, A.Th.M. \& Beenakkers, A.M.Th. (1980). Flight muscle development in Locusta migratoria: effects of implantation of corpora allata on the attainment of metabolic enzymes activities. General and Comparative Endocrinology 41: 477-486. https:// doi.org/10.1016/0016-6480(80)90052-0

Villalobos-Sambucaro, M.J., Nouzova, M., Ramirez, C.E., Alzugaray, M.E., Fernandez-Lima, F., Ronderos, J.R. \& Noriega, F.G. (2020). The juvenile hormone described in Rhondius prolixus by Wigglesworth is juvenile hormone III skipped bisepoxide. Scientific Reports 10: 3091. https:// doi.org/10.1038/s41598-020-59495-1

Webb, G., Miller, P. \& Peters, B. (2012). Pyriproxyfen for the Control of Australian Salt-Marsh Mosquito, Aedes vigilax. Journal of the American Mosquito Control Association 28: 50-52. https://doi.org/10.2987/11-6139.1

Westerlund, S.A. \& Hoffmann, K.H. (2004). Rapid quantification of juvenile hormones and their metabolites in insect haemolymph by liquid chromatography-mass spectrometry (LC-MS). Analytical and Bioanalytical Chemistry 379: 540-543. https://doi.org/10.1007/s00216-004-2598-x

Wijayaratne, L.K.W. Fields, P.G. \& Arthur, F.H. (2012). Effect of methoprene on the progeny production of Tribolium castaneum (Coleoptera: Tenebrionidae). Pest Management Science 68: 217-224. https://doi.org/10.1002/ps.2247

Wilson, T.G. \& Turner, C. (1992). Molecular analysis of methoprene-tolerant, a gene in Drosophila involved in resistance to juvenile hormone analog insect growth regulators. In: Molecular Mechanisms of Insecticide Resistance, Mullin C.A \& Scott J.G. (editors). Washington: American Chemical Society, pp. 8-99.

Wu, S.J., Zhang, J.Z. \& Ma, M. (1987). Monitoring the effects of juvenile hormones and 20-hydroxyecdysone on yolk polypeptide production of Drasophila melanogaster with enzyme immunoassay. Physiological Entomology 12: 355-361. https://doi.org/10.1111/j.1365-3032.1987.tb00761.x

Wyatt, G.R. \& Davey, Kg. (1996). Cellular and molecular actions of juvenile hormone II. Roles of juvenile hormone in adult insects. Advance In Insect Physiology 26: 1-155. https:// doi.org/10.1016/S0065-2806(08)60030-2

Yamamoto, R., Hua Bai, Dolezal, A.G., Amdam, G. \& Tatar, M. (2013). Juvenile hormone regulation of Drasophila aging. BMC Biology 11: 85. https://doi.org/10.1186/1741-7007-1185

Yang, W.J., Xu, K.K., Shang, F., Dou, W. \& Wang, J.J. (2016). Identification and characterization of three $\mathrm{JH}$ genes from Bacterocera dorsalis (Diptera: Tephritidae). The Florida Entomologist 99: 648-657. https://doi.org/10.1653/024.099. 0411

Yin, C.M. (1994). Juvenile hormone III bisepoxide: New member of the insect juvenile hormone family. Zoological Studies 33: $237-245$. 